\title{
EVALUACIÓN CLÍNICA Y RADIOGRÁFICA DE UNA PASTA ACUOSA DE HIDRÓXIDO DE CALCIO - IODOFORMO EN EL TRATAMIENTO DE PIEZAS PRIMARIAS NECRÓTICAS (SEGUIMIENTO DE TRES MESES)
}

\author{
CORRAL-PEÑAFIEL DAVID* \\ VÉLEZ-LEÓN MARÍA**
}

Recibido: 15/11/2015

Aceptado: 18/11/2015

\section{RESUMEN}

Objetivo: Evaluar la eficacia de una pasta acuosa de hidróxido de calcio - iodoformo en el tratamiento de piezas primarias necróticas (seguimiento de tres meses). Materiales y Métodos 28 piezas dentarias con necrosis pulpar pertenecientes a 25 niños de 3 a 8 años que asistieron a la clínica odontológica de la Universidad Católica De Cuenca recibieron tratamientos de pulpectomías con preparación mecánica con limas y química con hipoclorito de sodio y fueron obturadas con pasta acuosa de hidróxido de calcio iodoformado. Se hicieron controles clínico radiográficos durante 90 días de las variables dolor, fístula, movilidad y radiolucidez periapical e interradicular. Resultados: A los 90 días, todas las piezas se presentaron asintomáticas, el $93.1 \%$ presentaron ausencia de movilidad dental, el $79.3 \%$ presentaron ausencia de lesión periapical; 17 piezas se encontraban subobturadas. Conclusiones: En esta muestra la obturación de pulpectomías en piezas primarias con necrosis realizada con pasta acuosa de hidróxido de calcio iodoformo fue exitosa clínica y radiográficamente a 90 días. Debería ampliarse el estudio a más largo plazo y compararlo con otras técnicas.

Palabras clave: Eficacia, lodoformo, Endodoncia, Odontopediatría

\section{ABSTRACT}

Aim: To evaluate the efficacy of a calcium hydroxide iodoform aqueous paste on the treatment of primary necrotic pieces ( 3 month follow-up). Materials and Methods: 28 dental pieces with pulp necrosis belonging to 25 children between 3 and 8 years old who attended the odonthologic clinic of the Catholic University of Cuenca and received pulpectomies through mechanical preparation with files and chemical treatment with sodium hypoclorite and were sealed with calcium hydroxide iodoform aqueous paste. Clinical radiographic controls of the variables: pain, fistula, mobility, and periapical and interrradicular radiodiolucency were carried out during 90 days. After 90 days all the pieces were asymptomatic, $93.1 \%$ had absence of tooth mobiity, $79.3 \%$ had no periapical lesion, 17 pieces were found to be subobturated. Conclusions: This sudy shows that the primary pieces with necrosis which underwent pulpectomy obturation treated with calcium hydroxide iodoform aqueous paste were succesful both clinically and radiographically after 90 days. This study should hbe extended and compared to other techniques

Keywords: Efficiency, lodoform, Endodontic, Pediatric Dentistry.

\section{INTRODUCCIÓN}

La terapia pulpar ayuda a preservar la pieza dental hasta el momento de su exfoliación cuando la pulpa dental en piezas deciduas se ve comprometida por caries, traumas dentales u otros factores. Uno de los factores que pueden coadyuvar con el éxito es el material usado en la obturación, para evitar la presencia de microorganismos y asegurar un sellado hermético de los conductos sin herir a la pieza sucesora o evitando afectar a la salud del paciente ${ }^{1}$.

El lodoformo es una sustancia reabsorbible, radiopaca, de fácil manipulación y no produce cambios en el germen dental de la pieza sucesora. Tiene propiedades analgésicas, y efectos antibacterianos, se presenta como un sólido en forma de cristales hexagonales amarillos de color y sabor característicos ${ }^{2}$. Se considera que es tolerado en el periápice aún en grandes sobreobturaciones ${ }^{3}$.Una de las presentaciones utilizadas desde hace casi un siglo es en forma de pasta combinada con hidróxido de calcio y clorofenol alcanforado ${ }^{3}$.

El hidróxido de calcio en una solución saturada, de disociación iónica con liberación de iones hidroxilo $(\mathrm{OH}-)$ e

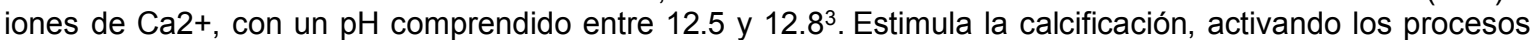
reparativos por activación osteoblástica; al aumentar el $\mathrm{pH}$ en los tejidos adyacentes ${ }^{1}$. Es antibacteriano debido a que las condiciones del $\mathrm{pH}$, bajan la concentración de iones de Hidrógeno e inhiben la actividad enzimática de las bacterias. Puede esterilizar hasta un $88 \%$ de los conductos radiculares ${ }^{1}$.

El uso de vehículos aceitosos para transportar el hidróxido de calcio genera una baja solubilidad y baja capacidad de difusión en los tejidos, en las que la disociación iónica no ocurre, por lo que el efecto del hidróxido de calcio sería nulo. Por ello algunos autores consideran que utilizar un aceite como vehículo sería un error. Químicamente es imposible medir el pH de un aceite, puesto que no permiten la disociación de iones $\mathrm{H}+\mathrm{y} \mathrm{OH}$ ,confirmando la incompatibilidad del $\mathrm{Ca}(\mathrm{OH})^{1}$.

El objetivo de este estudio fue evaluar la acción clínica y radiográfica de una pasta acuosa de hidróxido de calcio iodoformo en pulpectomías de piezas primarias necróticas en el primer trimestre postratamiento.

* Odontólogo egresado, Universidad Católica de Cuenca. Ecuador

** Docente Especialista en Odontopediatria, Universidad Católica de Cuenca.Ecuador 


\section{MATERIALES Y MÉTODOS}

Diseño: Prospectivo experimental descriptivo.

Muestra: Se incluyeron todos los niños de ambos sexos entre 3 y 8 años cuyos padres brindaron consentimiento y que presentaban molares primarios con diagnóstico de necrosis pulpar. Fueron excluidos los niños con incapacidad física, mental y motora y las piezas dentarias con reabsorción de más de 1/3 radicular.

La muestra quedó constituida por 28 piezas dentarias pertenecientes a 25 niños que asistieron a la clínica odontológica de la Universidad Católica de Cuenca.

Todos los tratamientos fueron realizados por un único operador, estudiante de odontología.

Métodos: Los tratamientos se realizaron siguiendo el protocolo que se desarrolla a continuación y fueron evaluados clínica y radiográficamente a los 30,60 y 90 días. Las variables consideradas por presencia 0 ausencia fueron: dolor, fistula, movilidad, lesión periapical y/o interrradicular y ensanchamiento del espacio periapical. La información para la obtención de los resultados se cargó en el programa SPSS 18.

\section{Protocolo clínico:}

1. Anestesia infiltrativa con jeringa tipo Carpule y lidocaína al $2 \%$.

2. Aislamiento absoluto del campo operatorio con dique de goma y arco de Young

3. Acceso a la cámara pulpar con la pieza de alta velocidad, y con una fresa con forma de bola

4. Cuando la cámara pulpar está expuesta y exenta de caries, se irriga con hipoclorito desodio al $2.5 \%$ y se seca con torundas de algodón estéril.

5. Localización de la entrada a los conductos (Fig.1). Las limas de tipo K se introducen en el conducto.

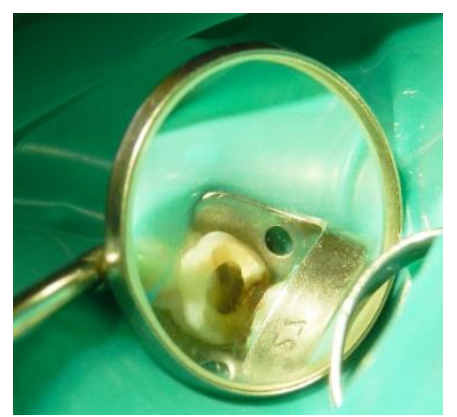

Fig. 1 Localización de los conductos radiculares Fuente: Propia

6. Una vez localizado el ápice radicular en la radiografía, se procede a instrumentar a $1 \mathrm{~mm}$ del ápice. Se deben instrumentar los conductos con las limas de tres medidas más que con las que se inició la instrumentación

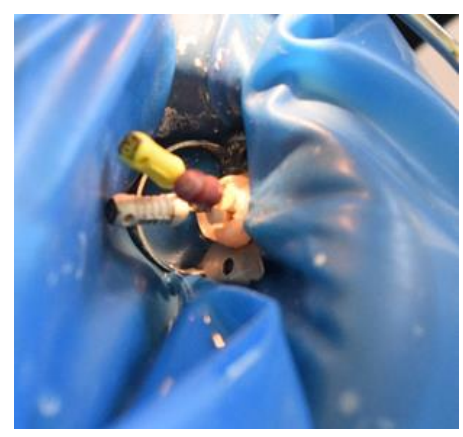

(Fig.2) Instrumentación de los conductos dentales con limas k Flex, Fuente: Propia

Entre una lima y otra de calibre más amplio, se irriga el conducto con hipoclorito de sodio al 2\% (Fig.3). 


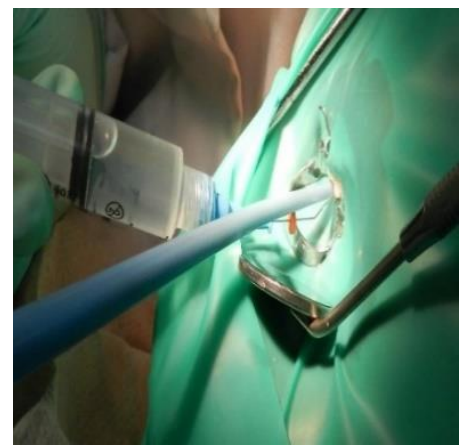

Fig. 3 Irrigación de los conductos radiculares con hipoclorito de sodio al 1\%, Fuente: Propia

Finalizada la instrumentación, se seca con puntas de papel del mismo número al de la última lima con que se instrumentó (Fig.4).

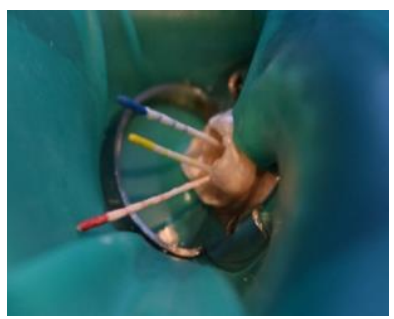

Fig. 4 Secado de los conductos radiculares con puntas absorbentes de papel VDM. Fuente: Propia

7. Obturación: se utiliza pasta lodoformada preparada con dos partes de hidróxido de calcio (2.8g), una parte de iodoformo $(1.4 \mathrm{~g})$ y $3 \mathrm{~cm} 3$ de agua destilada en una loseta estéril. Se lleva a los conductos con limas, colocando un tope medio milímetro menos que la medida utilizada para instrumentar, hasta que se rebase el material. Posteriormente se presiona nuevamente el material presente en la cámara pulpar, hacia la entrada de los conductos en los que se quiere lograr una mejor obturación. obturación (Fig.5).

Se limpia la cavidad de excedentes y se coloca una base de óxido de zinc y eugenol y se rehabilita la pieza con corona de acero (Fig.6)

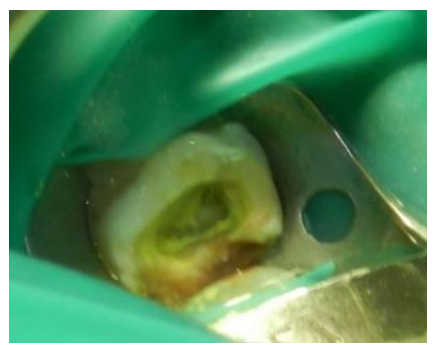

Fig 5. Secado de los conductos radiculares con puntas

Se limpia la cavidad de excedentes y se coloca una base de óxido de zinc y eugenol y se rehabilita la pieza con corona de acero (Fig. 6) ${ }^{3}$.

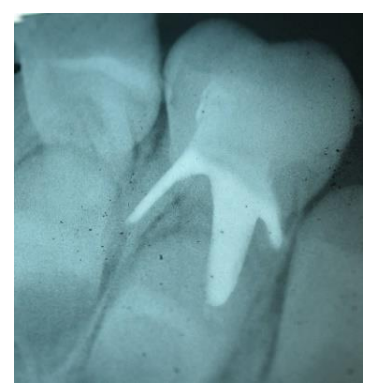

Fig. 6 Imagen radiográfica donde se puede notar la radiopacidad de la pasta lodoformada en los conductos radiculares 


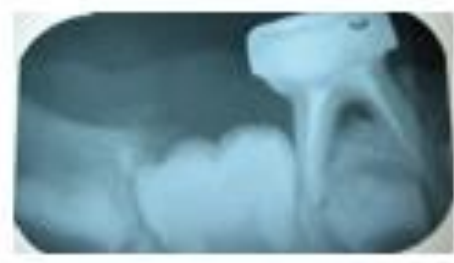

Caso cllnico 1. 1er Mes.

Fuente: David Francisco Corral Pehanel

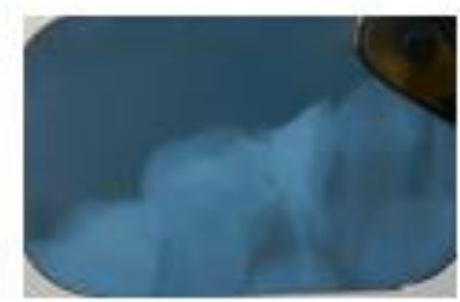

Caso cllnico 2. 1er Mes.

Fuente: David Francisco Corra! Pehiaflel

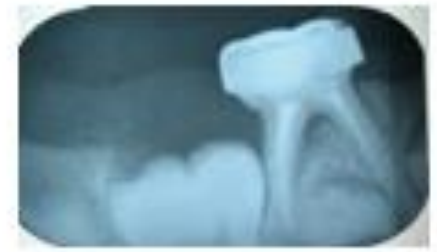

Caso clinico 1. 2 do Mes.

Fuente: David Franciaco Corral Peflafiel

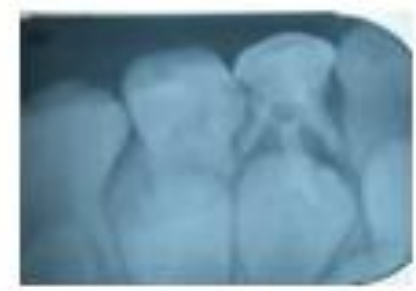

Caso clinico 2. 2 do Mes.

Fuente: David Francisco Corral Pefianel

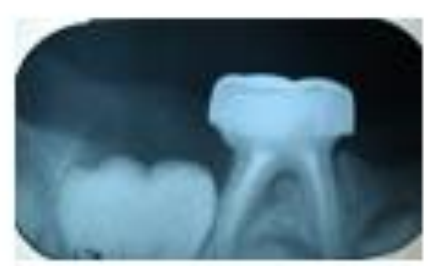

Caso clinico 1. 3er Mes. Fuente: David Francisco Corral Pehlafiel

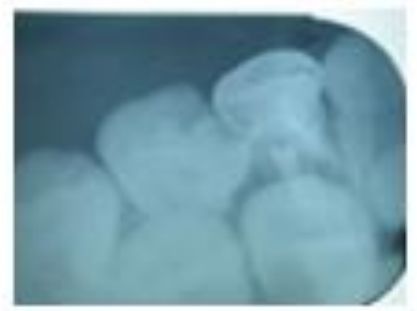

Caso clinico 2. 3er Mes.

Fuente: David Francisco Corral Pethanel

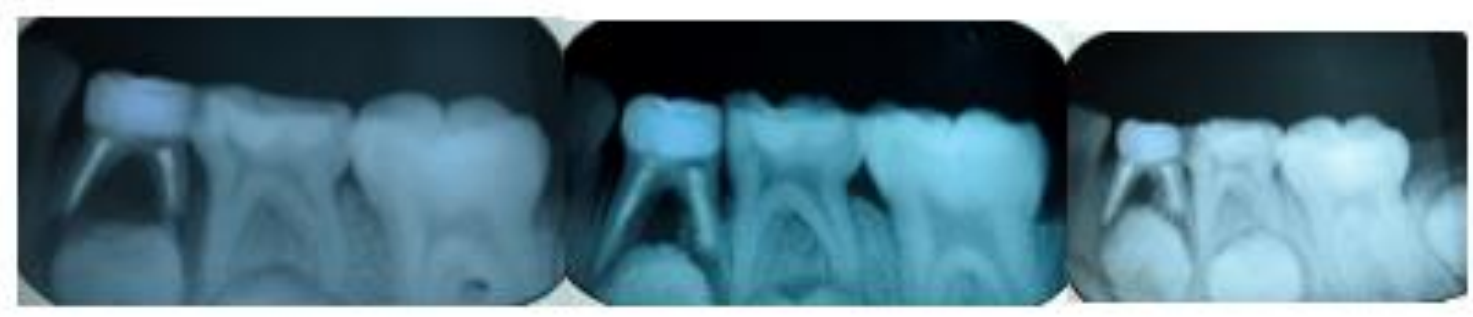

Caso cllnico 3. 1er Mes. Fuente: David Francisco Corral Pehlafiel

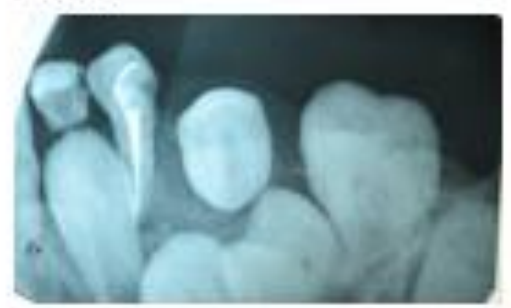

Caso cilnico 4. 1er Mes.

Fuente: David Francisco Corral Pefianel
Caso clinico 3. 2do Mes. Fuente: David Francisco Corral Pehiaflel

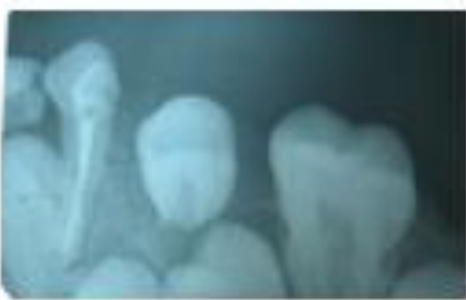

Caso clinico 4. 2 do Mes. Fuente: David Francleco Corral Pehaflel
Caso cllnico 3. 3er Mes.

Fuente: David Franclsco Corral Pehlanel

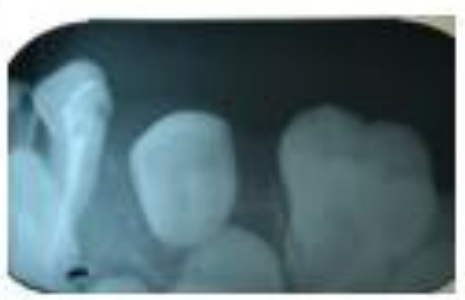

Caso clinico 4. 3er Mes.

Fuente: David Francisco Corral Pehanel

Fig 7. Serie de Casos 


\section{RESULTADOS}

\section{Evaluación Clínica}

El 96,6\% de las piezas tratadas no presentaron dolor ni fístula en ninguno de los controles.

El $17,2 \%$ de las piezas tratadas mostraron movilidad dental grado I a los 30 días, el 6,9\% persistía a los 60 días y el $3,4 \%$ a los 90 días.

\section{Evaluación Radiográfica}

A los 30 días el 96,6\% de los pacientes atendidos presentaron signos de lesión periapical, a los 60 días, el $44,8 \%$ aun persistía y finalmente a los 90 días de control solo el 17,2 \% de las piezas tratadas presentaban aun lesión periapical y/o interrradicular.

A los 30 días el 96,6\% de pacientes atendidos presentaban ensanchamiento del espacio periodontal, a los 60 días el $20,7 \%$ y a los 90 días, solo el $10,3 \%$.

Se observó que 17 piezas a los 90 días mostraron reabsorción del material de obturación.

Tabla 1.

\begin{tabular}{cccccccc}
\hline Acción Clínica Y Radiográfica De La Pasta lodoformada En Los Tratamientos De Pulpectomía De Piezas Necróticas. & \multicolumn{3}{c}{$\begin{array}{c}\text { 30 días } \\
\end{array}$} & \multicolumn{2}{c}{60 días } & \multicolumn{2}{c}{90 días } \\
& si & no & si & no & si & no \\
\hline Dolor & 0 & 28 & 0 & 28 & 0 & 28 \\
Fistula & 0 & 28 & 0 & 28 & 0 & 28 \\
Movilidad Dental & 5 & 23 & 2 & 26 & 1 & 27 \\
Lesión periapical & 28 & 0 & 13 & 15 & 5 & 23 \\
Ensanchamiento de ligamento periapical & 28 & 0 & 6 & 22 & 3 & 25 \\
\hline
\end{tabular}

\section{DISCUSIÓN}

La terapia pulpar en piezas primarias presenta múltiples dificultadas que no han sido consideradas en este estudio. Tanto la conducta del niño como la complejidad anatómica de los conductos radiculares de las piezas primarias que dificultan la preparación mecánica son algunos de los motivos por los cuales hasta la fecha no hay consenso en cuál sería el tratamiento ideal que evite tratamientos costosos e invasivos tales como las exodoncias y mantenimiento de los espacios.

La presencia de lesiones periapicales en necrosis pulpar de piezas temporales induce a la búsqueda de materiales para la obturación canalicular que brinden respuestas favorables para la reparación. Las publicaciones reportan algunos resultados exitosos con productos comerciales disponibles en el mercado internacional.

Marín Rivera, utilizando la pasta comercial de hidróxido de calcio iodoformada Vitapex®, en piezas vitales y no vitales reportó a 120 días ausencia de dolor y reparación ósea en la mayoría de los casos coincidiendo con los resultados de este estudio a 90 días.

Barja-Fidalgo ${ }^{4}$, halló resultados radiográficos favorables a 6 meses utilizando 2 pastas comerciales con iodoformo una con hidróxido de calcio y otra con óxido de zinc (Vitapex® y Metapex®) por lo que puede estimarse que a mayor plazo los tratamientos de este estudio continúen siendo exitosos.

En un trabajo presentado por Jha Mihir ${ }^{1}$ se reporta ausencia de dolor en un $96 \%$ de piezas obturadas hasta el ápice dental en los primeros 30 días, en éste estudio no hubo hallazgos de dolor en el primer mes.

Uno de los factores que debería considerarse al elegir el material de obturación es el tiempo requerido para el recambio dentario. Las pastas con base de hidróxido de calcio son rápidamente reabsorbibles mientras que las que contienen óxido de cinc son lentamente reabsorbibles. El hidróxido de calcio se reabsorbe rápidamente en presencia de secreciones. También es necesario evaluar la acción del hapteno que podría tener el iodoformo.

\section{CONCLUSIONES}

En esta muestra la obturación de pulpectomías en piezas primarias con necrosis realizada con pasta acuosa de hidróxido de calcio iodoformo fue exitosa clínica y radiográficamente a 90 días. Debería ampliarse el estudio a más largo plazo y compararlo con otras técnicas. 


\section{REFERENCIAS BIBLIOGRÁFICAS}

1. Seale NS, Col JA. Vital pulp therapy for the primary dentition. General Dentistry. 2010 (32) pp 194-200

2. Ladines MC, Medicación Intraconducto con Hidróxido de Calcio, Yodoformo Paramonoclorofenol Alcanforado en dientes necróticos. Universidad de Guayaquil. Facultad de Odontología. 2013 (pp 23-33.) Disponible en: http://repositorio.ug.edu.ec/handle/redug/3651

3. Marianella MR. Pulpectomía Utilizando Pasta Premezclada De Hidróxido De Calcio Con Yodoformo, Como Material De Obturación, En Piezas Temporales. Universidad Latinoamericana De Ciencia Y Tecnología Facultad De Odontología. 2010. [Citado 24 Abr 2015] pp 12-38. Disponible en http://eprints.uanl.mx/2182/1/1080175104.pdf

4. Cabrera Dávila MJ. Eficacia Del Uso De Hidróxido De Calcio Vs Óxido De Zinc Eugenol En Tratamientos De Pulpotomías En Niños De 5 A 11 Años De Edad [tesis]. Ecuador. Universidad Católica De Santiago De Guayaquil Facultad De Ciencia Medicas Carrera De Odontología. 2010. (pp 49-54). Disponible en: http://repositorio.ucsg.edu.ec/handle/3317/906

5. Liewehr DHP y FR. Estructura y funciones del complejo dentinopulpar. En Stephen Cohen KM. Vías de la pulpa. Séptima edición. Madrid: Elsevier. 2007. (pp 469-522).

6. Mendoza Zambrano G. Índice De Eficacia En Pulpotomías Y Pulpectomías En Pacientes Pediátricos De 5 A 7 Años De Edad Atendidos En La Clínica Odontológica De La Universidad Católica Santiago De Guayaquil. UCSG. 2011. (marzo 25 2015) disponible en: http://repositorio.ucsg.edu.ec:8080/handle/123456789/927

7. Joe H. Camp AF. Endodoncia pediátrica: tratamiento endodóntico en la dentición temporal y permanente joven. En Stephen Cohen KH. Vías de la pulpa. Décima edición. Madrid: Elsevier. 2011. (pp 808-857.).

8. Assed, S; Freitas, A, C; Da Silva, L, A; Filheo, P. En Leonardo MR. Tratamiento Endodóntico en Dientes Temporales. Endodoncia. Tratamiento de Conductos Radiculares. Sao Paulo: Artes Médicas. 2005. (151-208).

9. Fuks AB. Guideline on pulp therapy for primary and young permanent teeth. AAPD, 2014, (36): (pp 242250).

10. Sociedad Española De Odontopediatría. [Internet] Madrid, Sociedad Española de Odontopediatría y Academia Americana de Odontopediatría, (Agosto 30, 2012; Abril 25, 2015) disponible en http://www.odontologiapediatrica.com/pulpa

11. Bailleul-Forestier I, Molla M, Verloes A, \& Berdal A. The genetic basis of inherited anomalies of the teeth: Part 1: Clinical and molecular aspects of non-syndromic dental disorders. European Journal of Medical Genetics, Patologías pulpares y tratamientos endodónticos en dientes primarios. Estudio de casos base de datos. 2014. (51). (pp 273-291).

12. Ashraf Fouad LL. Efectos de la caries y los tratamientos dentales sobre la pulpa. En Stephen Cohen K. Vías De La Pulpa. Séptima Edición. Madrid. Elsevier. 2007. (pp 524-574)

13. Fuks AB. Tratamiento pulpar para la dentición primaria. En Pinkham JR. Odontología pediátrica. Tercera edición. McGraw: Hill Interamericana. (pp 368-383).

14. Muñante Cárdenas JL. Identificación De Microorganismos Anaerobios Facultativos Y Anaerobios Estrictos Frecuentes En Necrosis Pulpares. Universidad Nacional Mayor De San Marcos. 2009. (Pp 7-38). Disponible En: http://cybertesis.unmsm.edu.pe/handle/cybertesis/1732

15. Ash M. Dientes deciduos o primarios En Wheeler. Anatomía, fisiología y oclusión dentales de Wheeler. D.F: Nueva Editorial Interamericana, 2014. (pp 46-82).

16. González Rodríguez E. Diagnóstico y tratamiento pulpar en dentición temporal. En J. Boj. Odontopediatría. Primera Edición. Barcelona: Masson, 2011. (pp 173-183)

17. James L. Guttmann, J. Craig Baumgartner, Alan H. Gluskin, Gary R. Hartwell, MS, \& Richard E. Walton, American Board of Endodontics Pulpal and Periapical Diagnostic Terminology. JOE. 2014; (35): pp 1658-1674

18. Leonardo MR. Tratamiento endodóntico de dientes temporales con vitalidad pulpar. En Bezerra L. Tratado de Odontopediatría Sao Paulo: Amolca. (pp 613-662).

19. Solís Abril DG. Selección De Fármacos Utilizados En Pulpotomías De Dientes Temporales [Tesis]. Ecuador. Universidad De Guayaquil Facultad Piloto De Odontología. 2012. (Pp 14-19) disponible en: http://repositorio.ug.edu.ec/handle/redug/2980 